$44^{\text {th }}$ AIAA/ASME/ASCE/AHS/ASC Structures, Structural Dynamics, and Materials Conference AIAA-2003-1536 April 7-10, 2003, Norfolk, VA

\title{
CALCULATION OF NON-BONDED FORCES DUE TO SLIDING OF BUNDLED CARBON NANOTUBES
}

\author{
Frankland, S. J. V., Bandorawalla, T. ${ }^{\dagger}$, and Gates, T. S. ${ }^{*}$ \\ NASA Langley Research Center \\ Hampton, VA 23681
}

\begin{abstract}
$\underline{\text { ABSTRACT }}$
An important consideration for load transfer in bundles of single-walled carbon nanotubes is the nonbonded (van der Waals) forces between the nanotubes and their effect on axial sliding of the nanotubes relative to each other. In this research, the non-bonded forces in a bundle of seven hexagonally packed $(10,10)$ single-walled carbon nanotubes are represented as an axial force applied to the central nanotube. A simple model, based on momentum balance, is developed to describe the velocity response of the central nanotube to the applied force. The model is verified by comparing its velocity predictions with molecular dynamics simulations that were performed on the bundle with different force histories applied to the central nanotube. The model was found to quantitatively predict the nanotube velocities obtained from the molecular dynamics simulations. Both the model and the simulations predict a threshold force at which the nanotube releases from the bundle. This force converts to a shear yield strength of 10.5-11.0 MPa for $(10,10)$ nanotubes in a bundle.
\end{abstract}

\section{$\underline{\text { INTRODUCTION }}$}

Single-walled carbon nanotubes (SWNT) are nanoscale hollow cylinders made of a single layer of graphite. ${ }^{1}$ The synthesis of SWNTs often results in the formation of bundles consisting of hexagonal arrays of nanotubes. ${ }^{2}$ From the standpoint of

\footnotetext{
${ }^{*}$ Staff Scientist, National Institute of Aerospace

${ }^{\dagger}$ Post-doctoral Associate, Old Dominion University

¥ Senior Materials Research Engineer, Mechanics and Durability

Branch, AIAA Associate Fellow

Copyright (C) 2003 by the American Institute of Aeronautics and

Astronautics, Inc. No copyright is asserted in the United States under

Title 17, U.S. Code. The U.S. Government has a royalty-free license to exercise all rights under the copyright claimed herein for

Governmental Purposes. All other rights are reserved by the copyright owner.
}

mechanical performance, bundled SWNTs may be the material form of carbon nanotubes that holds the most promise for bulk nanotube composites. However, nanotubes are not continuous over great lengths and individual nanotubes in the bundle can vary in length such that any number of nanotubes can be shorter than the length of the bundle. Hence, if a tensile force was to be applied to such a bundle of discontinuous SWNTs, the individual nanotubes would have a tendency to slide past each other. This sliding will occur unless the non-bonded (van der Waals) forces between nanotubes are high enough to resist relative motion. Therefore, it is important to quantify the non-bonded forces between bundled nanotubes and understanding how these forces affect the relative sliding of nanotubes.

Although there are currently no direct experiments on the relative sliding of nanotubes in bundles recorded in the literature, there are several examples of experiments and models which seek to describe the strength of the non-bonded interactions of nanotubes. Two recent laboratory experiments have addressed sliding between the shells of multiwalled carbon nanotubes (MWNT). ${ }^{3,4}$ In the first experiment, upper bounds on static and dynamic interlayer friction were estimated to be 0.66 and $0.43 \mathrm{MPa}$, respectively, from measurements on the contact area of nested nanotube shells with high-resolution transmission electron microscopy shells that were used in conjunction with a model for the van der Waals energy. ${ }^{3}$ In the second experiment, shear interaction strengths of 0.08 and $0.3 \mathrm{MPa}$ were obtained for two different MWNTs from displacement and force curves measured during sliding and pulling out of nested nanotube shells by a mechanical-loading stage inside a scanning electron microscope. ${ }^{4}$ In this experiment the static and dynamic shear interaction strengths were equivalent.

Modeling efforts have also addressed the non-bonded interactions between nanotubes ${ }^{5}$ and of nanotubes with polymers. ${ }^{6-9}$ One molecular dynamics simulation study has indicated that shear load transfer in SWNTs bundles with an added degree of twist between the nanotubes may be significantly higher than load transfer between nanotubes in an untwisted bundle. ${ }^{5}$ The authors have used molecular dynamics simulations to estimate the force required to pull the 
nanotube through a polyethylene composite in which the embedded nanotube was either covalently bonded to the polymer or had non-bonded van der Waals interactions (represented by the Lennard-Jones potential) with the polymer. ${ }^{6-8}$ The results of this work have shown that at even low grafting densities of chemical bonds, such as those introduced through defects, covalent bonding of the polymer to a nanotube can make a large difference in the shear yield strength. ${ }^{6}$ The frictional behavior of the nanotube sliding through the polymer matrix beyond the yield point has also been described with a Bingham fluid-type model which was used to characterize the threshold stress and the interfacial viscosity obtained from the simulation. ${ }^{7,8}$ Another simulation effort on polymer-nanotube composites used molecular mechanics to compare the shear yield strengths of nanotubes in several different helicalwrapped polymer chains. ${ }^{9}$

The present work on the mechanical performance of SWNT bundles is motivated by the current lack of experimental data and validated analyses that address the relative sliding behavior of nanotubes in bundles. The objective of the research is to quantify the effect that the net force on a nanotube in a bundle, arising from non-bonded interactions with its neighboring nanotubes, has on the axial sliding of nanotubes. The approach taken is to develop a simple model, based on momentum balance, that is verified with molecular dynamics (MD) simulations. As a representative case, the system under study consists of seven continuous SWNTs arranged in a hexagonal-shaped array, without supporting matrix. First, several different force histories are applied to the central nanotube in the bundle, and the system response is simulated by MD. Next, the momentum model is developed to include the non-bonded interactions between nanotubes and the force histories from MD. The momentum model is then parameterized with non-bonded force information from an MD simulation. Velocity profiles of the central nanotube are subsequently predicted from the momentum model and compared to those obtained from MD simulation. Finally, a shear yield strength estimate is made from the MD simulation results for nanotube pull-out in bundles in comparison to the earlier work $^{6}$ on nanotube pull-out in polymer composites.

\section{MOLECULAR DYNAMICS SIMULATIONS}

Molecular dynamics simulations model the interactions of a collection of particles at the atomistic scale. MD simulates path-dependent processes by solving the equations of motion for all the particles. Theoretical description of the method is beyond the scope of this paper; the reader is referred to Raabe ${ }^{10}$ for these details.

MD simulations were performed on a system of seven $(10,10)$ single-walled carbon nanotubes arranged in a hexagonal close-packed form (Fig. 1), where $(10,10)$ refers to the structural arrangement of the carbon atoms and is often referred to as the "armchair" configuration. ${ }^{1}$ The nanotubes are $13.6 \AA$ in diameter, a size typically observed in experiments done on single-walled carbon nanotubes. ${ }^{1}$

In the simulations, the chemical bonding in the nanotubes was modeled with the carbon potential developed by Brenner, et al. ${ }^{11}$ The non-bonding interactions between nanotubes were accounted for by using the Lennard-Jones potential with the parameters, $\sigma=3.35 \AA$ and $\varepsilon=51.2 \mathrm{~K}^{12}$ The equations of motion were integrated with a thirdorder Gear predictor-corrector algorithm, at a time step of 1 fs. Periodic boundary conditions were applied in the axial direction. The starting structure in the periodic structure contained seven flexible tubes of length $106 \AA$, separated by $3.2 \AA$. The axial length of the periodically repeating volume was held constant during the simulation. The initial configuration was partially equilibrated with a generalized Langevin thermostat at $300 \mathrm{~K}$ for 2000 steps integrated at $2 \mathrm{fs}$ time steps. The equilibration of the system was then continued at $300 \mathrm{~K}$ at a $1 \mathrm{fs}$ time step for 20000 time steps by controlling the temperature with velocity rescaling. Afterward, the outer six nanotubes were then fixed in place and the equilibrated configuration was the starting point of the MD simulations to be described.

In each MD simulation, force was applied to each atom of the inner nanotube, and the velocity of every atom in the inner nanotube was monitored. Three different kinds of loading profiles were used that are referred to as Type I, II, and III, herein. Type I was a single step increase in force $\Delta F$, at $3 \mathrm{ps}$, after which the force was held constant. Type II was an incrementally increasing force on the inner nanotube applied at a constant rate of $\Delta F$ after every time interval $\Delta t$, after an initial application of $(0.27 \mathrm{nN})$. Type III was an incrementally increasing force that was applied at a constant rate (after the initial application of $0.27 \mathrm{nN}$ ), as in Type II, and then incrementally decreased at the same rate back to a zero-valued applied force. The details of the MD simulations cases are summarized in Table I. 
$44^{\text {th }}$ AIAA/ASME/ASCE/AHS/ASC Structures, Structural Dynamics, and Materials Conference AIAA-2003-1536 April 7-10, 2003, Norfolk, VA

Table 1. Summary of MD simulations

\begin{tabular}{cccc}
\hline \hline Case & Type & $\Delta F(\mathrm{nN})$ & $\Delta t(\mathrm{ps})$ \\
\hline 1 & II & 0.0413 & 3.0 \\
2 & II & 0.0138 & 3.0 \\
3 & II & 0.0689 & 6.0 \\
4 & II & 0.0689 & 3.0 \\
5 & II & 0.138 & 3.0 \\
6 & II & 0.0138 & 0.1 \\
7 & I & 0.606 & -- \\
8 & III & 0.0689 & 3.0 \\
\hline \hline
\end{tabular}

\section{MOMENTUM MODEL FOR SWNT INTERACTION}

Consider the SWNT bundle as shown in Fig. 1. The balance of momentum for each atom, denoted by the subscript $i$, in the inner nanotube is given by

$$
\left(\mathbf{F}_{i}^{e}+\mathbf{F}_{i}\right) d t=m_{i} d \mathbf{V}_{i}
$$

where $\mathbf{F}_{i}^{e}$ is the force, as a result of all possible bonded and non-bonded atomic interactions, $\mathbf{F}_{i}$ is the external applied force, $t$ is the time, $m_{i}$ is the atomic mass, and $\mathbf{V}_{i}$ is the velocity. Bold face uppercase fonts represent vector quantities. The force $\mathbf{F}_{i}^{e}$ includes the forces from the bonded interactions within the inner nanotube $\mathbf{F}_{i}^{B}$, and the forces from the non-bonded interactions from the surrounding six nanotubes $\mathbf{F}_{i}^{N B}$, such that $\mathbf{F}_{i}^{e}=\mathbf{F}_{i}^{B}+\mathbf{F}_{i}^{N B}$. A summation of Eq. (1) over all $n$ atoms in the inner nanotube yields

$$
\sum_{i=1}^{n}\left(\mathbf{F}_{i}^{e}+\mathbf{F}_{i}\right) d t=\sum_{i=1}^{n} m_{i} d \mathbf{V}_{i}
$$

where

$$
\sum_{i=1}^{n} \mathbf{F}_{i}^{e}=\sum_{i=1}^{n} \mathbf{F}_{i}^{B}+\sum_{i=1}^{n} \mathbf{F}_{i}^{N B}
$$

However

$$
\sum_{i=1}^{n} \mathbf{F}_{i}^{B}=0
$$

because the force on atom $i$, from atom $j$, is equal and opposite to the force on atom $j$, from atom $i$, by Newton's third law. Equation (2), therefore, reduces to

$$
\left(\mathbf{F}^{N B}+\mathbf{F}\right) d t=m n d \mathbf{V}
$$

where

$$
\begin{aligned}
& \mathbf{F}^{N B}=\sum_{i=1}^{n} \mathbf{F}_{i}^{N B}, \mathbf{F}=\sum_{i=1}^{n} \mathbf{F}_{i}, \text { and } \\
& d \mathbf{V}=\frac{1}{n} \sum_{i=1}^{n} d \mathbf{V}_{i}
\end{aligned}
$$

and $m$ is the mass of a carbon atom, $\mathbf{V}$ is the average velocity of the inner nanotube, $\mathbf{F}^{N B}$ is the net force on the central nanotube from the six neighbors, and $\mathbf{F}$ is the net externally applied force on the central nanotube.

Equation (5) involves vector quantities. Designating the nanotube axial direction as the Z-direction (Fig. 1) and integrating the Z-component of Eq. (5) from time $t_{1}$ to $t$ yields

$$
\int_{t_{1}}^{t}\left(F^{N B}+F\right) d t^{\prime}=m n\left[V(t)-V\left(t_{1}\right)\right]
$$

where $F^{N B}, V$, and $F$ are the Z-components of the quantities given by Eq. (6). The time derivative of Eq. (7) is

$$
F^{N B}(t)+F(t)=m n A(t)
$$

where $A$ is the average acceleration of the inner nanotube in the Z-direction. Equations (7) and (8) are simply the momentum balance in the Z-direction for the inner nanotube as a whole.

\section{$\underline{\text { RESULTS }}$}

This section describes how the momentum model was parameterized and applied to predict the velocity of the central nanotube for different applied force histories. Finally, a threshold force at which the nanotube releases from the bundle was calculated from the simulations.

\section{Momentum Model Calibration}

The momentum model was calibrated with the results from a molecular dynamics simulation involving the application of force at a constant rate (Case 1) after an initial application of force. The loading history is shown in Fig. 2 together with the average Zcomponent of velocity for the inner nanotube computed by molecular dynamics. This velocity is used in conjunction with Eq. (8) to obtain $F^{N B}(t)$ (Fig. $3)$. The force $F^{N B}$ results from van der Waals interactions of the central nanotube with the surrounding nanotubes, and is a function of the relative positions of the atoms in the inner nanotube, with respect to the surrounding nanotubes. Hence, for the purpose of making predictions with this model, it is convenient to express $F^{N B}$ as a function of the axial displacement $U$ of the inner nanotube. The 
$44^{\text {th }}$ AIAA/ASME/ASCE/AHS/ASC Structures, Structural Dynamics, and Materials Conference AIAA-2003-1536 April 7-10, 2003, Norfolk, VA

functional form of $F^{N B}(U)$ is shown in Fig. 4. Four points near zero displacement are omitted to improve the integration (see dotted line in Fig. 4 inset). The axial displacement $U(t)$ of the inner nanotube is directly available from the molecular dynamics results for Case 1 .

Some points need to be made about $F^{N B}(U)$ that is calculated by the method described herein. First, the force $F^{N B}$ can be calculated from the results of the other MD simulations given in Table 1 by using Eq. (8). Because the inner nanotube has $3 n$ degrees of freedom that respond differently to different $F(t)$, these calculations would result in different functional forms of $F^{N B}$ for each $F(t)$. Hence, although Fig. 4 is representative of the magnitude and periodicity of fluctuations in the non-bonded axial force on the inner nanotube, it is not unique. Second, to facilitate the interpretation of results, it is best to calculate $F^{N B}$ from a MD simulation with a relatively low axial velocity $V(t)$ because $F^{N B}(U)$ is calculated at discrete points and a low $V(t)$ would yield a monotonically increasing $U(t)$ with enough closely spaced points in $F^{N B}(U)$.

The force $F^{N B}(U)$ was also calculated by translating the central nanotube (as a rigid entity) with respect to the other six nanotubes (also rigid) and performing a molecular statics calculation. The molecular statics calculation over-predicted the magnitude of fluctuations in $F^{N B}(U)$ because the nanotubes do not translate as rigid entities in MD and significant local configuration changes occur during nanotube translation. The over-prediction resulted whether the starting configuration was an ideal configuration obtained from purely geometric considerations or an equilibrated structure from molecular dynamics (without an externally applied force).

Momentum Model Verification

To make velocity predictions for an arbitrary force $F(t)$, as applied in the molecular dynamics simulations, it is convenient to recast Eq. (8) as two first-order ordinary differential equations given by

$$
\begin{aligned}
& m n \frac{d V}{d t}=F(t)+F^{N B}(U) \\
& \frac{d U}{d t}=V(t)
\end{aligned}
$$

Equation (9) is solved for $V(t)$ by using a multi-step solver in MATLAB ${ }^{13}$ for stiff ordinary differential equations. This solver uses a backward differentiation formula, also known as Gear's method.
The momentum model result for Case 1 which was used for calibration is shown in Fig. 2 (dotted line). The nanotube velocity predicted from the momentum balance model agrees with the molecular dynamics simulation to within $1 \AA /$ ps.

The results from seven different molecular dynamics simulations (Cases 2-8) are compared to the momentum model predictions in Figs. 5-11. For each case, the velocity of the central nanotube, obtained from the MD simulation, is compared with the predicted velocity from the momentum model, by using $F^{N B}(U)$ from Fig. 4 (Case 1) as input. A ramped force $F(t)$, with progressively increasing ramp rates, is applied in Cases 2-6 (Figs. 5-9, respectively). As the ramp rate increases, the nonbonded force on the inner nanotube $F^{N B}$ becomes negligible with respect to the applied force $F(t)$; that is, $F^{N B}<<F$. Hence, at higher force ramp rates of $0.01 \mathrm{nN} / \mathrm{ps}$ to $0.1 \mathrm{nN} / \mathrm{ps}$ (Cases 3-6), the motion of the inner nanotube is dictated largely by its inertial response to $F(t)$, and there is better agreement between the momentum model and the corresponding MD results. The maximum deviation in these cases (Cases 3-6) between the velocities from the momentum model and the MD simulation ranges from $2-4 \AA / p s$ or $3-12 \%$ of the maximum velocity in the MD simulation. At the lower ramp rate of 4.6 $\mathrm{x} 10^{-3} \mathrm{nN} / \mathrm{ps}$ (Case 2), $F^{N B}$ plays a critical role in determining the velocity response of the inner nanotube. The maximum deviation between the velocity from the momentum model and the MD simulation in Case 2 is $3.5 \AA / p s$ or $15 \%$ of the maximum MD simulation velocity.

As a final check on the momentum model, it is compared to MD simulations in which a constant force is applied to the nanotube (Case 7; Fig. 10) and and in which a progressively increasing and then decreasing force (Case 8; Fig. 11). In the constant force case, the momentum balance model deviates $2.5 \AA / \mathrm{ps}$ or $22 \%$ of maximum velocity. This simulation reaches the lowest maximum nanotube velocity, and is therefore the most difficult case to match. In the case of increasing and decreasing force, the inertia of the inner nanotube results in a non-zero $V(t)$ even after the externally applied force has decreased to a value of zero. The agreement between the momentum balance model and the MD simulation is excellent regarding the shape of the curve. The error between the velocity from the momentum model and the MD simulation in Case 7 at the end of the simulation is $1 \AA / p s$ or $7 \%$ of the maximum velocity. 
$44^{\text {th }}$ AIAA/ASME/ASCE/AHS/ASC Structures, Structural Dynamics, and Materials Conference AIAA-2003-1536 April 7-10, 2003, Norfolk, VA

\section{Comparison to a Polymer-Nanotube Composite}

In previous work on polymer-nanotube composites, the shear yield strength, $\tau_{0}$, was calculated and used as the threshold stress for nanotube pull-out. ${ }^{6}$ By way of comparison to the polymer case, $\tau_{0}$ can be obtained from the simulations presented herein for nanotube bundles. Case 2 provides the most detailed information on $\tau_{0 .}$. The range of forces in the vicinity of the threshold force, before the nanotube begins to slide through the bundle, is $0.59-0.62 \mathrm{nN}$. This force value will vary slightly with the particular $(10,10)$ nanotube bundle configuration. The quantity $\tau_{0}$ is calculated from the threshold force at which the nanotube releases from the polymer. Dividing the threshold force through by the surface area of the inner nanotube results in $\tau_{0}=10.5-11.0 \mathrm{MPa}$. The surface area is calculated from the length of the nanotube and the radius of the nanotube, which includes half the van der Waals separation distance (assumed to be $3.35 \AA$ ). The value for $\tau_{0}$ will, however, be different for nanotubes of different radii. The range of $\tau_{0}$ values calculated herein is higher than the values reported for the sliding of nanotubes within multi-walled nanotube, at $0.3 \mathrm{MPa}^{4}$ The value is also higher than the $3 \mathrm{MPa}$ calculated for nanotubes in polyethylene. ${ }^{6}$

\section{CONCLUSIONS}

A simple model, based on momentum balance, has been developed to describe the relative sliding of nanotubes in a bundle as an axial force is applied to one of the nanotubes. The model incorporated the non-bonded interactions of a centrally located nanotube in a hexagonal bundle of seven nanotubes. The input for the non-bonded interactions was obtained from molecular dynamics simulations which used the Lennard-Jones potential to represent the van der Waals interactions between the nanotubes.

The model was verified by comparing its predictions to molecular dynamics simulation. In both the model and the simulations, an axial force was applied to the central nanotube with varying rate of increasing or decreasing force. The velocity of the nanotube under the different applied force histories was then compared with those predicted by the molecular dynamics simulation. At lower applied force loading rates, the non-bonded interactions play an important role in determining the sliding velocity of the nanotube. At higher applied force loading rates, the motion of the inner nanotube is dictated largely by its inertial response to the applied force.
In general, quantitative agreement was observed between the simulations and the model under the rates of forces applied, which translates to 0.01-0.1 $\mathrm{nN} / \mathrm{ps}$.

Both the simulations and the model also indicate that there is a force at which the nanotube releases from the bundle. From this force, the shear yield strength of nanotube in the bundle was found to be $\tau_{0}=10.5$ 11.0 MPa. This result is higher than the values observed for the relative sliding of nested shells of multi-walled carbon nanotubes and for single-walled nanotubes in polymer composites.

\section{$\underline{\text { ACKNOWLEDGMENTS }}$}

This research was partially supported by the National Aeronautics and Space Administration under NASA Contract No. NAS1-97046 while S. J. V. Frankland was in residence at ICASE, NASA Langley Research Center, Hampton, VA 23681.

\section{$\underline{\text { REFERENCES }}$}

1 Harris P. J. F., Carbon Nanotubes and Related Structures: New Materials For The 21st Century, Cambridge University Press, Cambridge, 1999.

2. Thess, A., Lee R., Nikolaev, P., Dai, H., Petit, P., Robert, J., Xu, C., Lee, Y. H., Kim, S. G., Rinzler, A. G., Colbert, D. T., Scuseria, G. E., Tomanek, D., Fischer, J. E., and Smalley R. E., "Crystalline Ropes of Metallic Carbon Nanotubes," Science, Vol. 273, 1996, pp. 483-487.

3. Cumings, J., and Zettl, A., "Low-Friction Nanoscale Linear Bearing Realized from Multiwall Carbon Nanotubes," Science, Vol. 289, 2000, pp. 602-604.

4. Yu, M. F., Yakobson, B. I., and Ruoff, R. S., "Controlled Sliding and Pullout of Nested Shells in Individual Multiwalled Carbon Nanotubes," J. Phys. Chem. B, Vol. 104, 2000, pp. 8764-8767.

5. Qian, D., Wagner, G. J., Liu, W. K., Yu, M. F., and Ruoff, R. S., "Mechanics of Carbon Nanotubes," Applied Mechanics Reviews, Vol. 55, 2002, pp. 495533. 
6. Frankland, S.J.V., Caglar, A., Brenner, D.W., and Griebel, M., "Molecular Simulation of the Influence of Chemical Cross-Links on the Shear Strength of Carbon Nanotube-Polymer Interfaces," J. Phys. Chem. B, Vol. 106, 2002, pp. 3046-3048.

7. Frankland, S. J. V., and Harik V. M., "Analysis of Carbon Nanotube Pull-Out From a Polymer Matrix," Surface Science, Vol. 525, 2003 pp. L103-108.

8. Frankland, S. J. V., and Harik V. M., "Simulation of Carbon Nanotube Pull-out When Bonded to a Polymer Matrix," Materials Research Society Symposium Proceedings, Vol. 740, 2002, I12.1.1-6.

9. Lordi, V., and Yao, N., "Molecular Mechanics of Binding in Carbon-Nanotube-Polymer Composites," J. Mater. Res., Vol. 15, 2000, pp. 2770-2779.

10. Raabe, D., Computational Materials Science: The Simulation of Materials Microstructures and Properties, Wiley, New York, 1998.

11. Brenner, D. W., Shenderova, O. A., Harrison, J. A., Stuart, S. J., Ni, B., and Sinnott, S. B., "Second generation reactive empirical bond order (REBO) potential energy expression for hydrocarbons," J. Phys C: Condensed Matter, Vol. 14, 2002, pp. 783

12. Allen, M. P., and Tildesley, D. J., Computer Simulation of Liquids, Clarendon Press, Oxford, 1987.

13. MATLAB Version 6, The MathWorks, Inc., 2000 . 
$44^{\text {th }}$ AIAA/ASME/ASCE/AHS/ASC Structures, Structural Dynamics, and Materials Conference April 7-10, 2003, Norfolk, VA

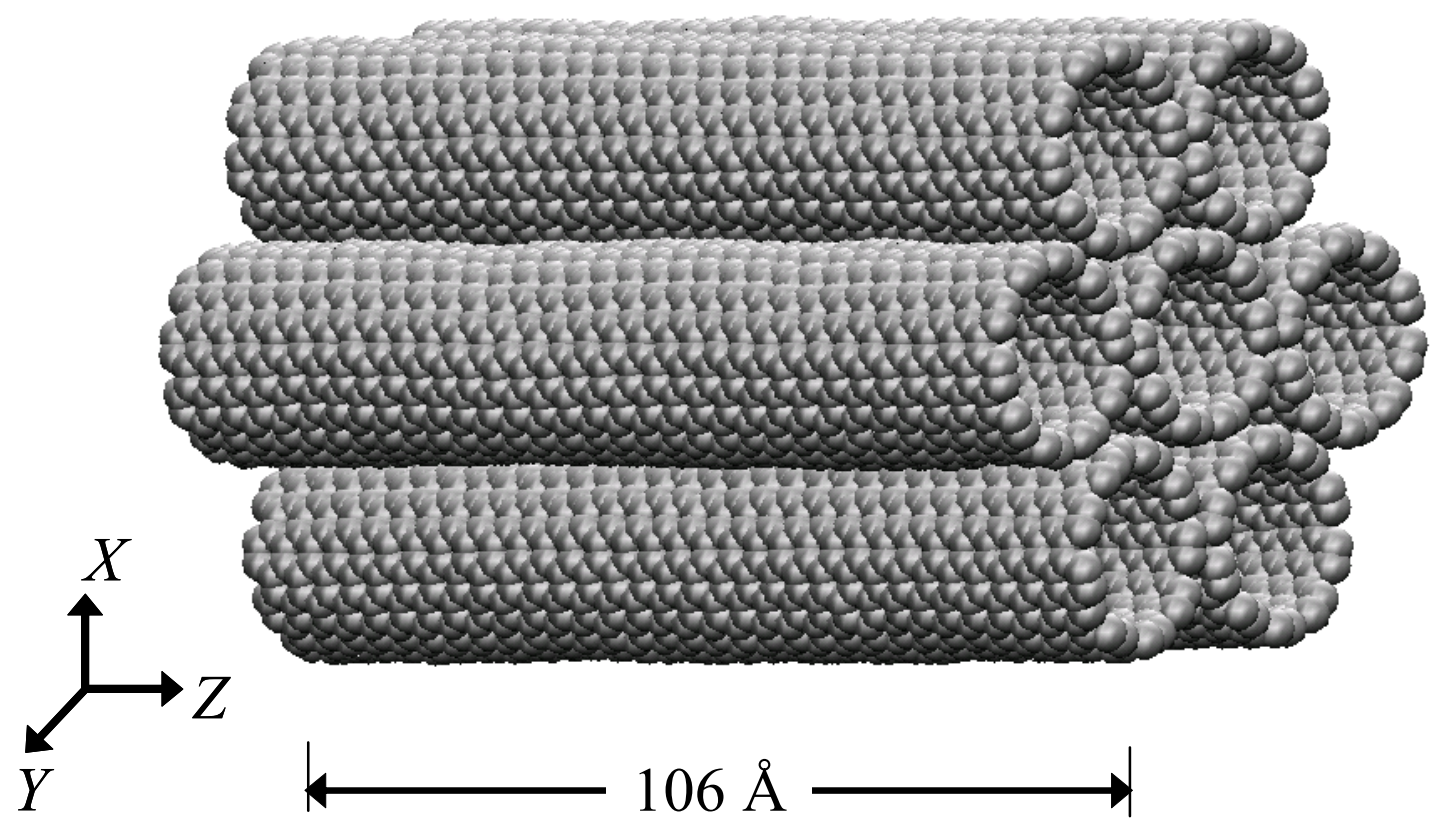

Fig. 1. System of seven nanotubes (not to scale)

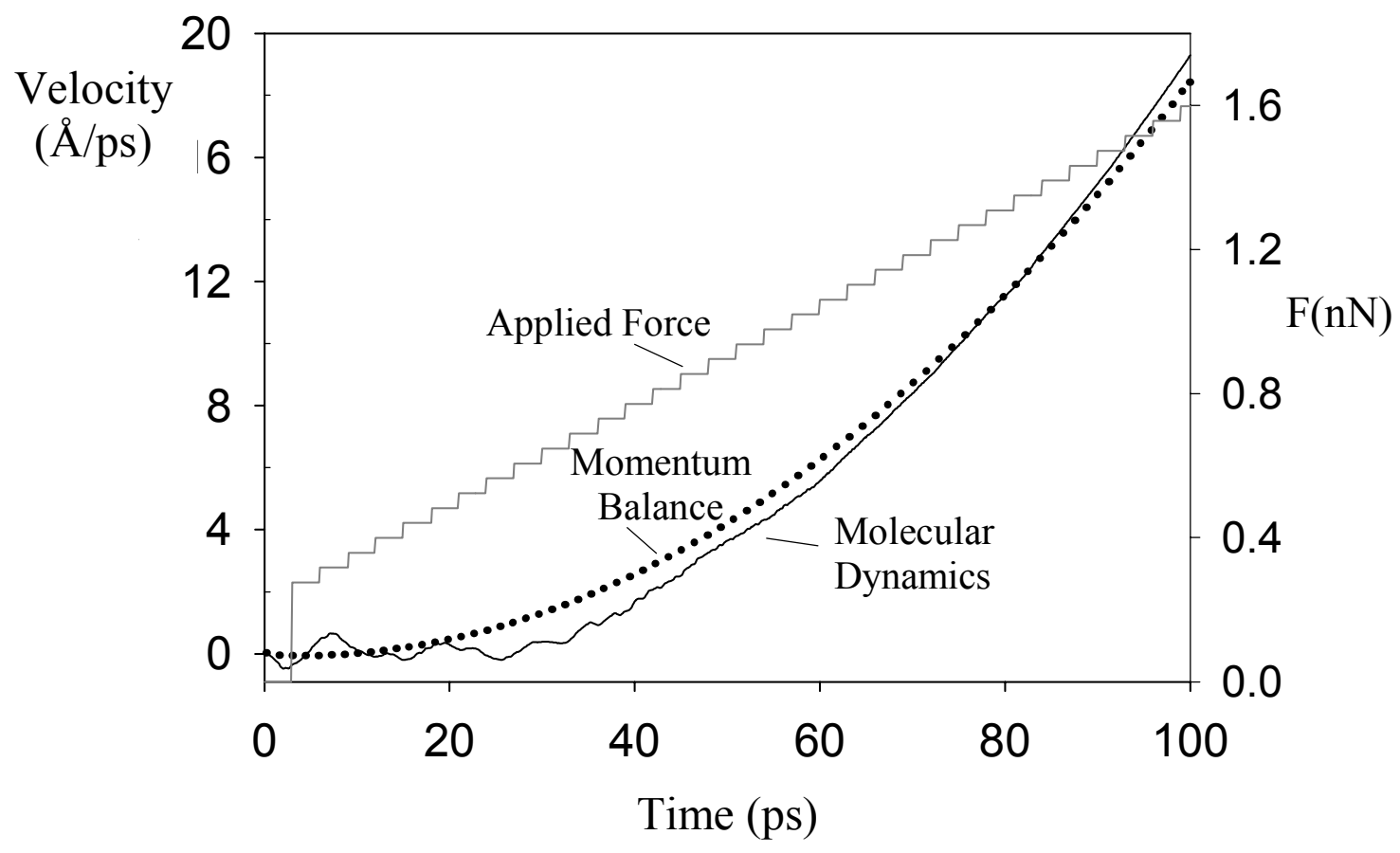

Fig 2. Nanotube average velocity. 


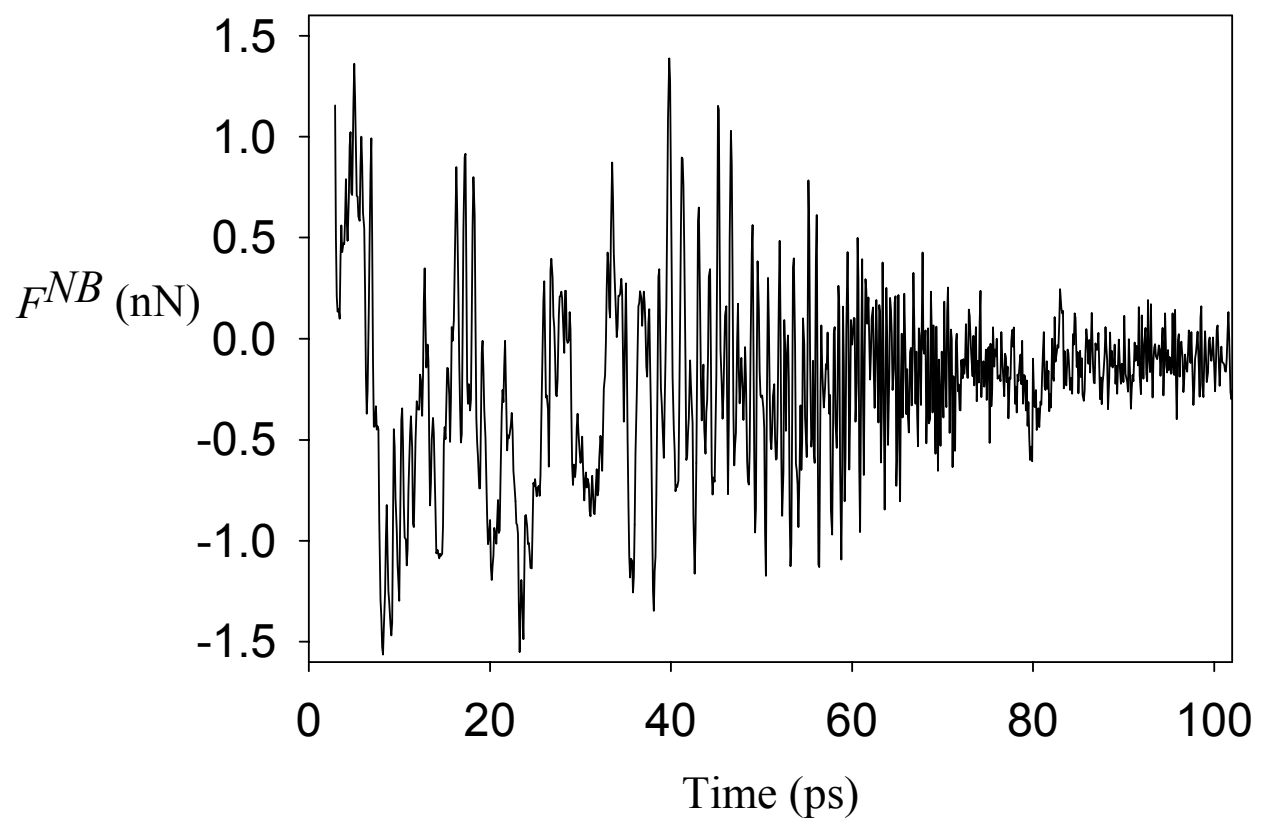

Fig. 3. Force exerted on inner nanotube due to non-bonded interactions with neighboring nanotubes

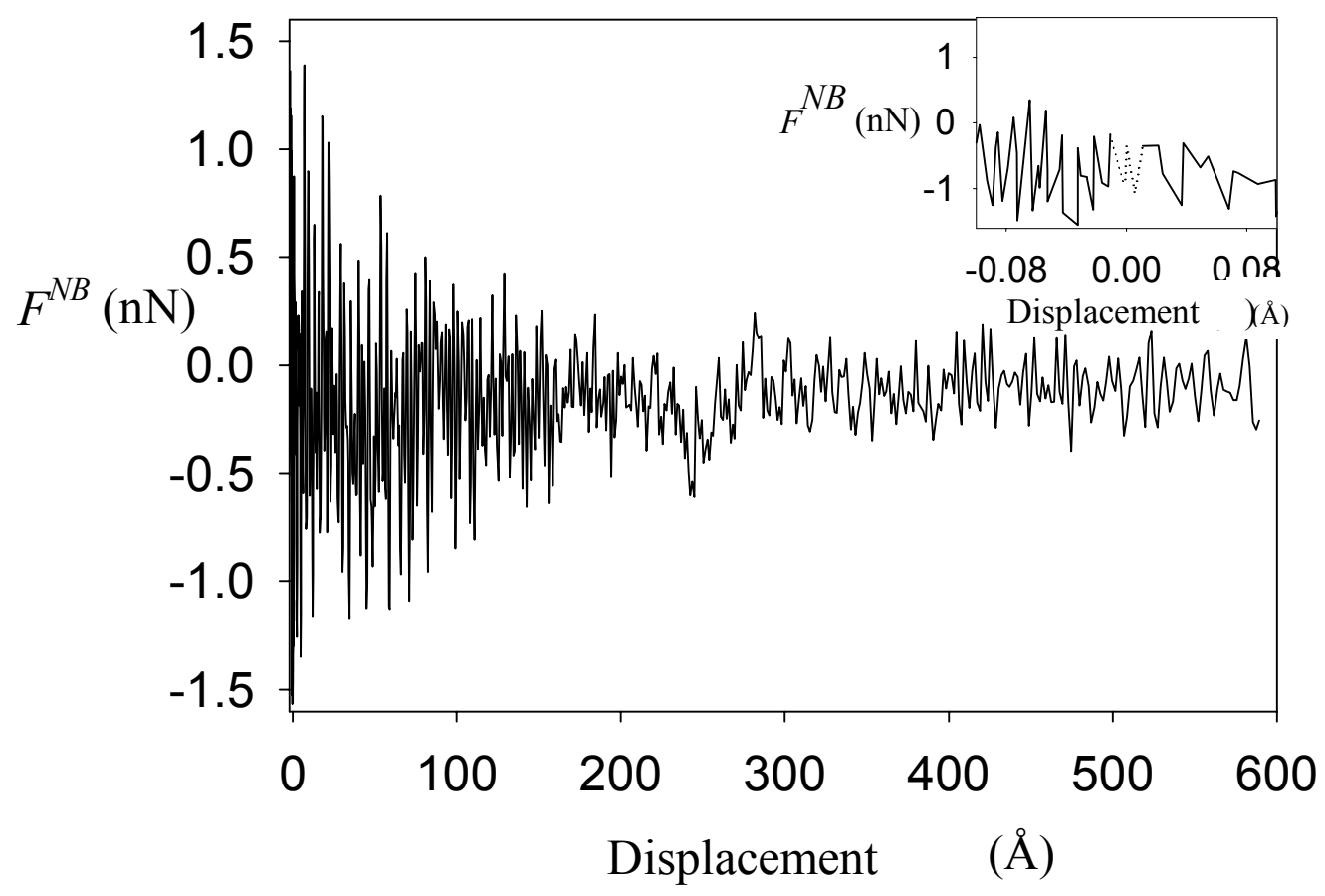

Fig. 4. Force exerted on inner nanotube due to non-bonded interactions with neighboring nanotubes (as a function of axial displacement of inner nanotube). Inset shows the points omitted in the fitting (dotted line). 


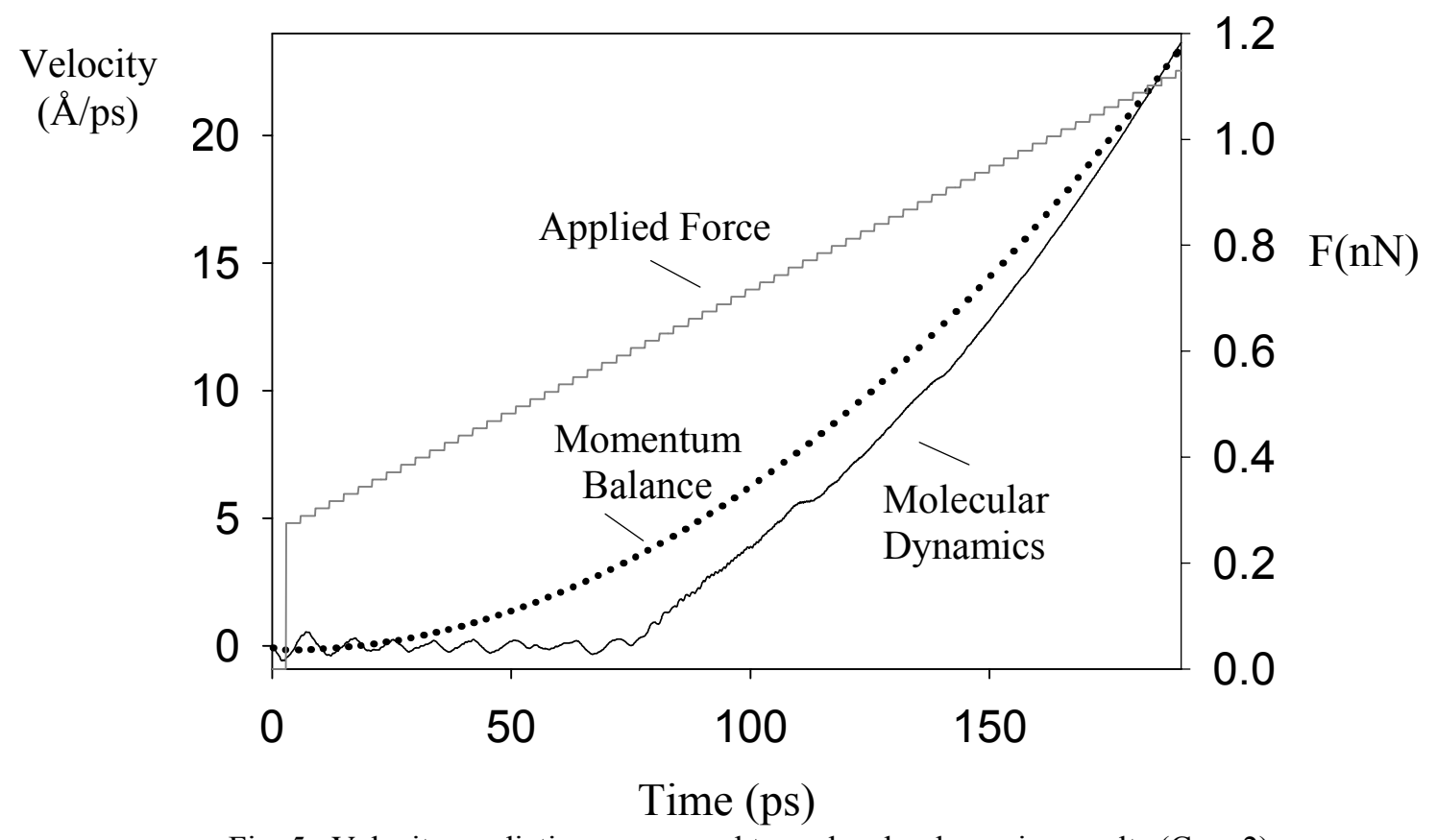

Fig. 5. Velocity predictions compared to molecular dynamics results (Case 2)

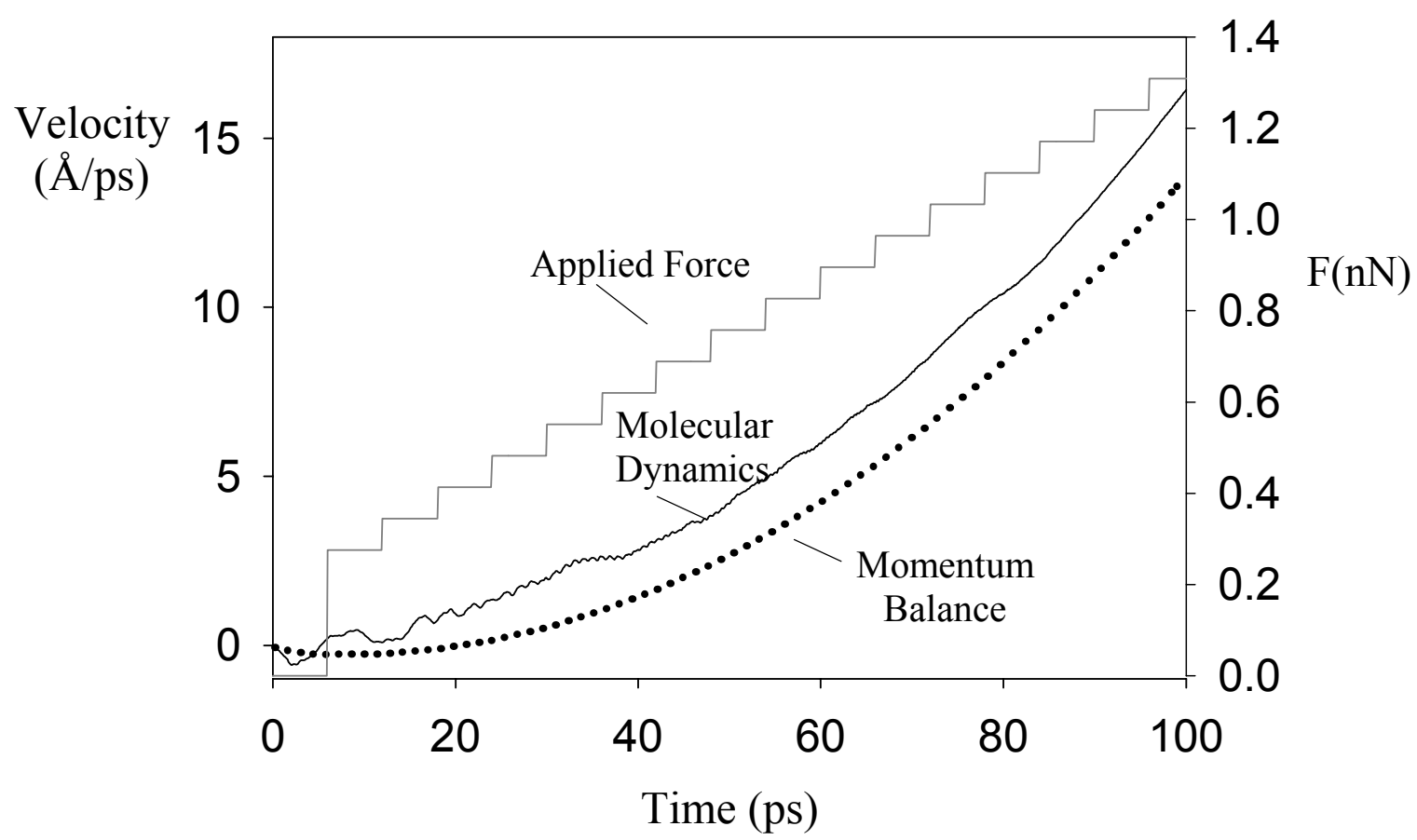

Fig. 6. Velocity predictions compared to molecular dynamics results (Case 3) 


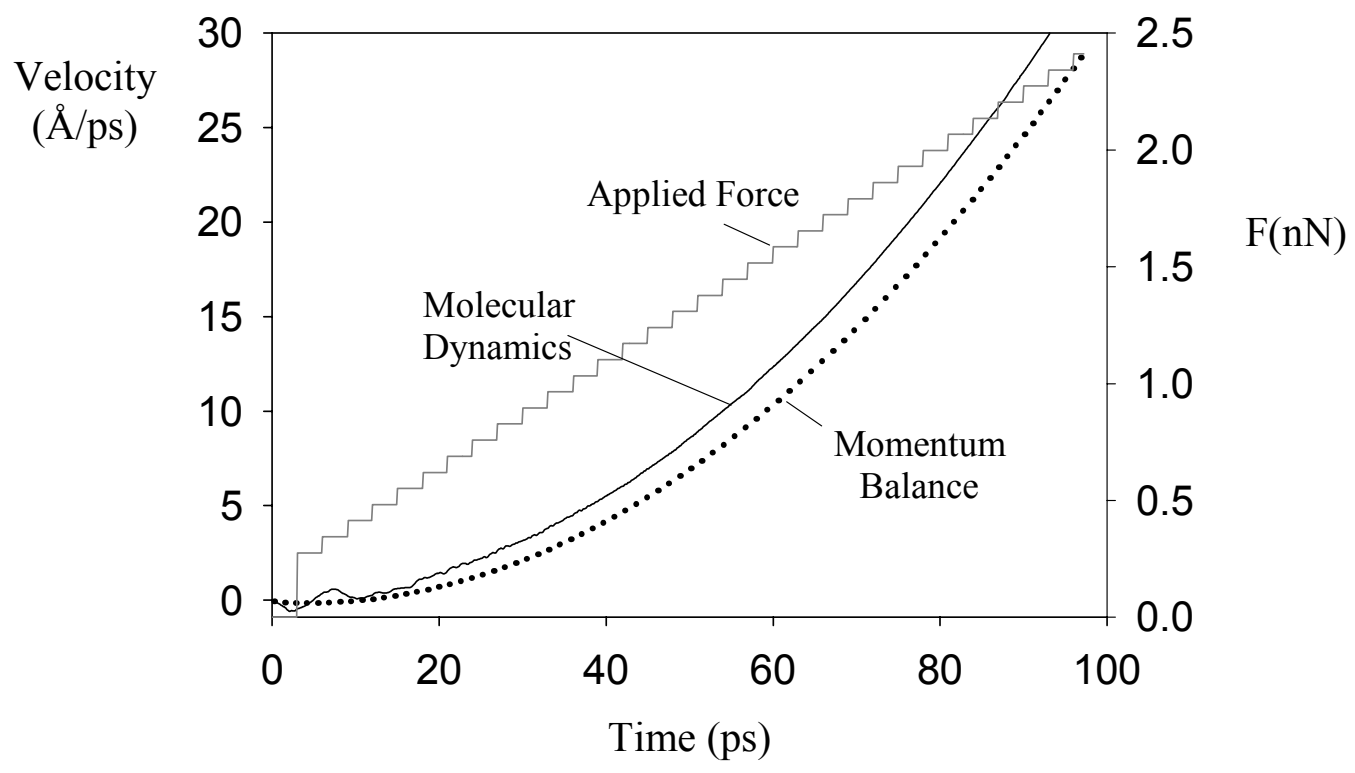

Fig. 7. Velocity predictions compared to molecular dynamics results (Case 4)

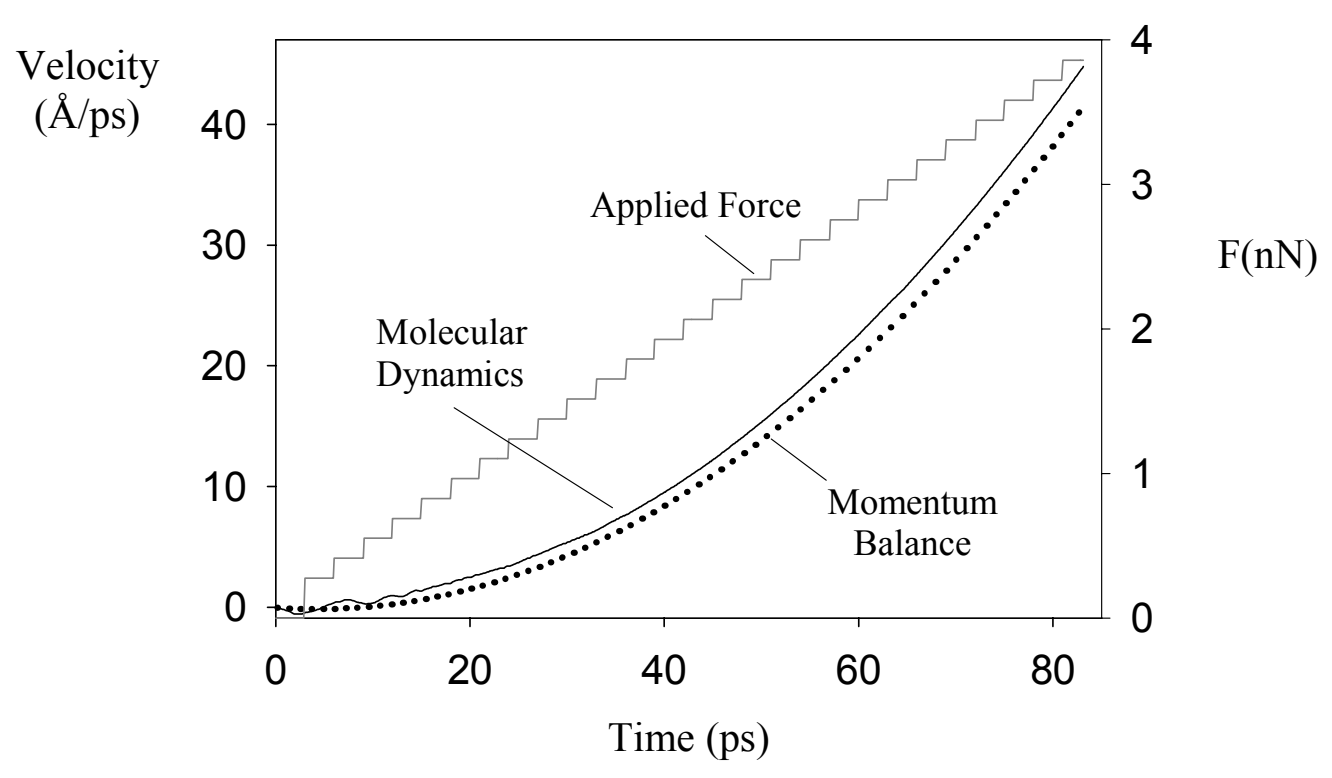

Fig. 8. Velocity predictions compared to molecular dynamics results (Case 5) 


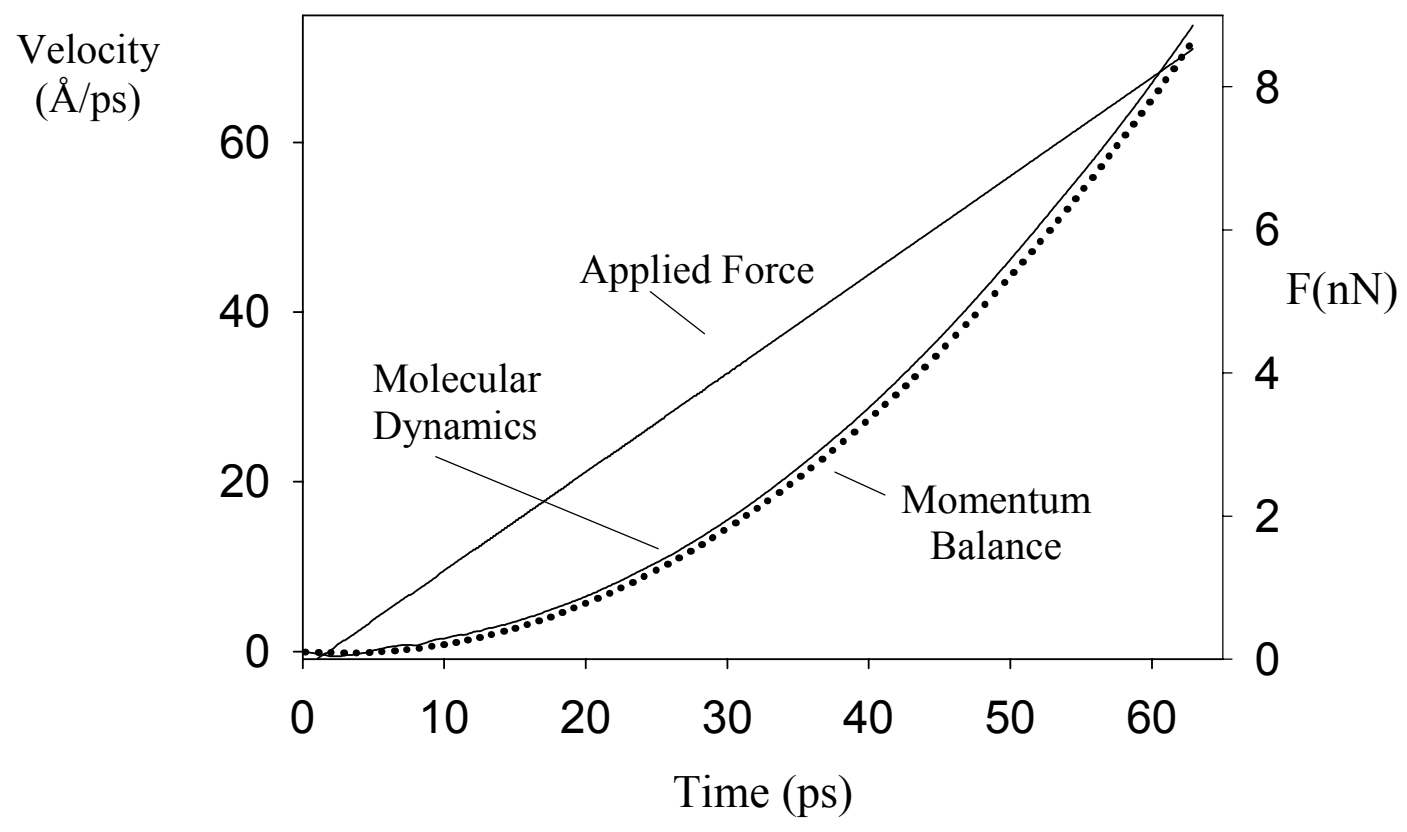

Fig. 9. Velocity predictions compared to molecular dynamics results (Case 6)

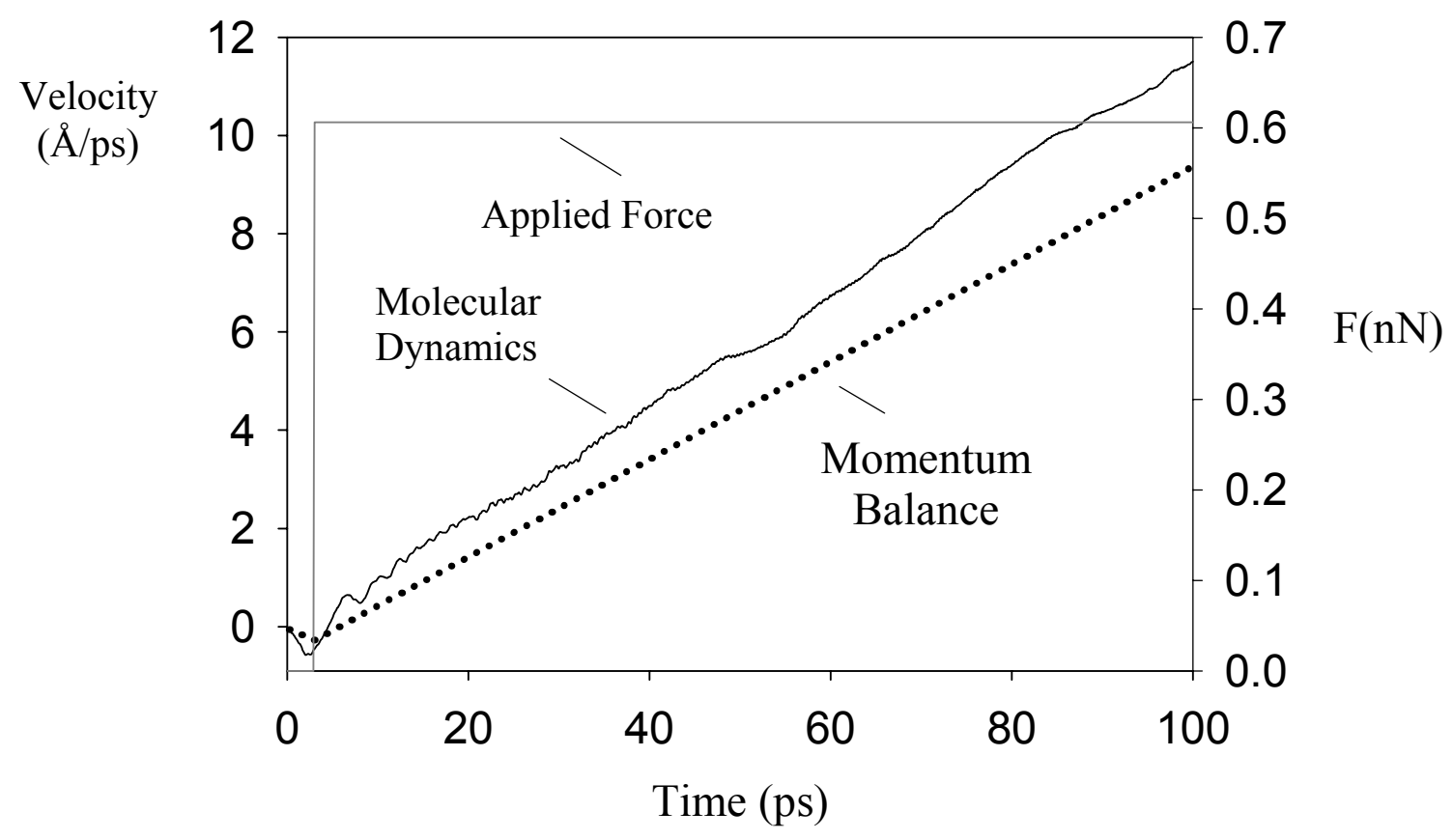

Fig. 10 Velocity predictions compared to molecular dynamics results (Case 7) 


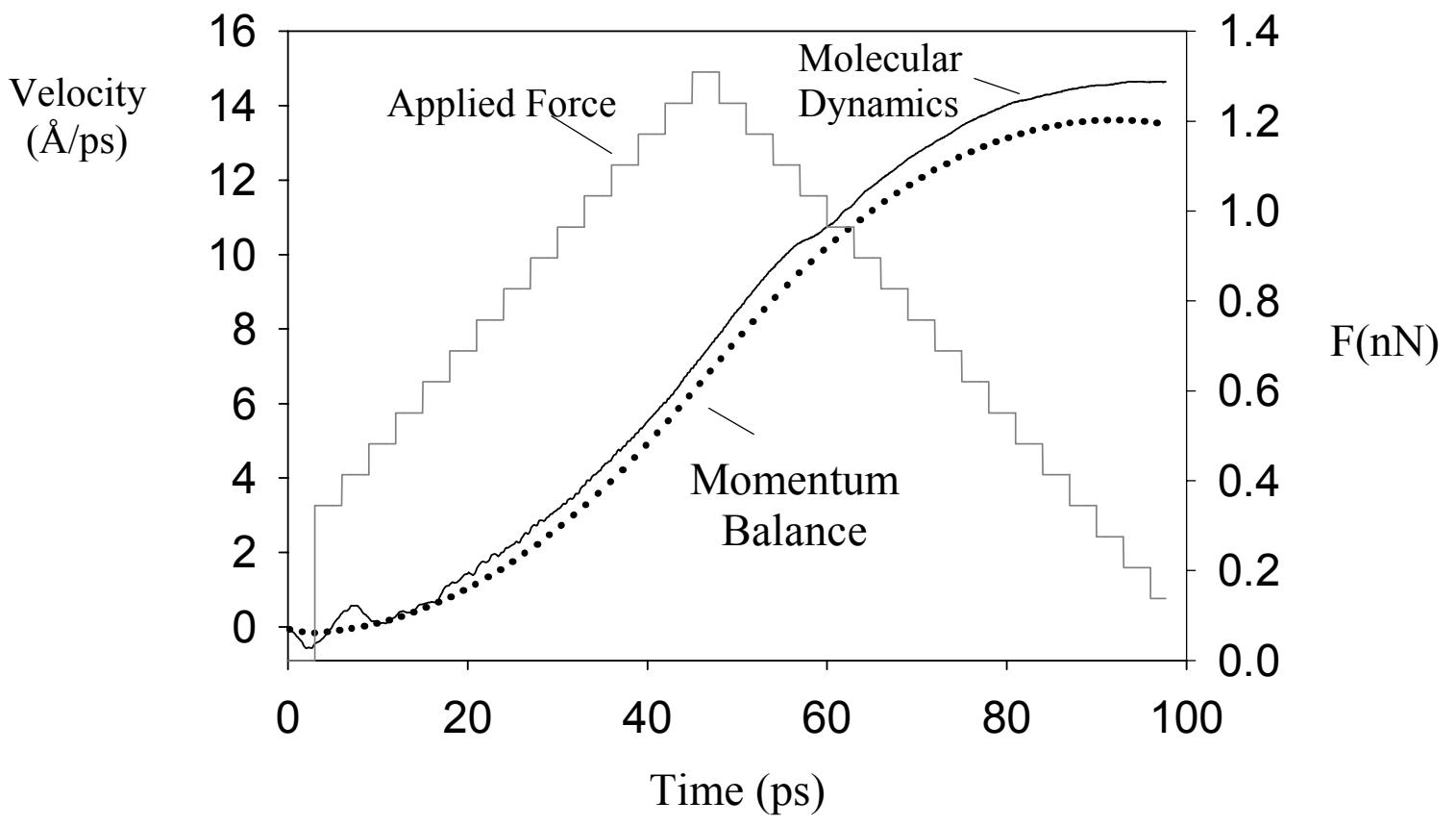

Fig. 11 Velocity predictions compared to molecular dynamics results (Case 8) 